\title{
Tolerância e crescimento de juvenis do robalo-flecha (Centropomus undecimalis) expostos a diferentes salinidades
}

\section{Tolerance and growth of juveniles of the common snook (Centropomus undecimalis) exposed to different salinities}

João Luiz Farias ${ }^{1 *}$; Carlos Antônio Zarzar²; Nelson Gomes da Silva-Neto²; Felipe dos Santos Silva²; Reginaldo Florêncio da Silva-Junior ${ }^{2} \&$ Ronaldo Olivera Cavalli ${ }^{1}$

${ }^{1}$ Programa de Pós-Graduação em Oceanografia, Universidade Federal de Pernambuco - UFPE

${ }^{2}$ Departamento de Pesca e Aquicultura, Universidade Federal Rural de Pernambuco - UFRPE

*E-mail: jeantanui@yahoo.com.br

Recebido: 7 de julho de 2017 / Aceito: 14 de julho de 2017/ Publicado: 9 de dezembro de 2017

Resumo Dois experimentos foram realizados para avaliar a sobrevivência e o desempenho de juvenis do robalo-flecha Centropomus undecimalis expostos a diferentes salinidades. No primeiro, grupos de 15 peixes com peso médio ( \pm DP) de 88,7 g $( \pm 23,6)$ foram transferidos diretamente da salinidade 15 para as salinidades $0,5,15,25$ e 35 . Amostras de sangue foram coletadas de três peixes por tratamento após $0,4,24$ e 96 h. Nenhuma mortalidade foi registrada. A concentração média de glicose $(114,3 \pm 32,0 \mathrm{mg} / \mathrm{dL})$ não diferiu entre as salinidades, enquanto a osmolaridade do plasma variou marginalmente, mas, após $96 \mathrm{~h}$, convergiu para 350-385 mOsm $/ \mathrm{kg}$. No segundo experimento não foram observas mortalidade e nem diferenças no nível glicêmico após 45 dias de criação nas salinidades $0,10,20,30$ e 40 . Não foram observadas diferenças no ganho de peso e na taxa de crescimento específico, mas o peso final foi maior na salinidade 30 do que nas salinidades 10 e 40 . A inexistência de alterações significativas em grande parte dos parâmetros mensurados neste estudo confirma a capacidade osmorregulatória de $C$. undecimalis $\mathrm{e}$, ao mesmo tempo, sugere a possibilidade de sua criação em ambientes com variadas condições de salinidade.

Palavras-Chave: estresse, osmorregulação; aquicultura, piscicultura marinha.

\begin{abstract}
Two trials were conducted to evaluate the survival and growth performance of juveniles of the common snook Centropomus undecimalis exposed to different salinities. In the first trial, groups of 15 fish with mean $( \pm$ SD) weight of $88.7 \mathrm{~g}( \pm 23.6)$ were transferred directly from salinity 15 to salinities $0,5,15,25$ and 35 . Blood samples were collected from three fish per treatment after $0,4,24$ and $96 \mathrm{~h}$. No mortality was recorded. The mean glucose concentration $(114.3 \pm 32.0 \mathrm{mg} / \mathrm{dL})$ was not different between salinities, whereas plasma osmolarity varied slightly, but after $96 \mathrm{~h}$ it converged to $350-385 \mathrm{mOsm} / \mathrm{kg}$. In the second trial no mortality and no differences in glycemic level were observed after rearing for 45 days at the salinities of $0,10,20,30$ and 40. No differences were observed in weight gain and specific growth rate, but the final weight was higher in fish reared at salinity 30 than at 10 and 40 . The lack of significant changes in most of the parameters measured here confirms the osmoregulatory capacity of $C$. undecimalis and, at the same time, suggests the possibility of its culture in areas with varying salinity.
\end{abstract}

Keywords: stress, osmoregulation, aquaculture, marine fish farming.

Trabalho financiado pela Coordenação de Aperfeiçoamento de Pessoal de Nível Superior - Capes, Fundação de Amparo à Ciência e Tecnologia do Estado de Pernambuco-Facepe. 


\section{Introdução}

O robalo-flecha ou camurim (Centropomus undecimalis) é um peixe marinho com alto potencial para a aquicultura (Cerqueira, 2004; Cavalli \& Hamilton, 2007; Alvarez-Lajonchère \& Tsuzuki, 2008; Winner et al., 2010), devido, principalmente, ao seu alto preço e grande demanda no mercado (Cerqueira, 2005; AlvarezLajonchère \& Tsuzuki, 2008; Cerqueira \& Tsuzuki, 2009; Liebl et al., 2016). Além disso, a tecnologia para a produção de juvenis dessa espécie em laboratório está razoavelmente bem desenvolvida (Alvarez-Lajonchère et al., 2002; Alvarez-Lajonchère \& Tsuzuki, 2008). Apesar disso, não há registros sobre a criação dessa espécie no Brasil (MPA, 2013), embora seja alternativa para os mais de 25.000 ha de viveiros utilizados unicamente na produção do camarão branco do Pacífico Litopenaeus vannamei.

O robalo-flecha se distribui naturalmente no Oceano Atlântico desde o sul do Brasil aos Estados Unidos (Rivas, 1986), onde realiza migrações entre as áreas costeiras e estuarinas (Figueiredo \& Menezes, 1980; Rivas, 1986; Cerqueira, 2005). Como esses ambientes costumam apresentar variações de salinidade, a sobrevivência e o crescimento dessa espécie podem ser afetados (Boeuf \& Payan, 2001; Sampaio \& Bianchini, 2002; Barman et al., 2005; Tsuzuki et al., 2007b). As espécies do gênero Centropomus são comumente encontradas em ambientes de baixa salinidade (Eskinazi, 1972; Gilmore et al., 1983; McMichael Jr. et al., 1989; Pérez-Pinzón \& Lutz, 1991; Pope et al., 2006), sendo, portanto, consideradas eurihalinas. Apesar disso, o conhecimento dos efeitos da salinidade sobre $C$. undecimalis ainda é limitado. Por exemplo, especificamente em relação à criação dessa espécie em cativeiro, Cerqueira \& Tsuzuki (2009) ressaltam a importância de se determinar com precisão os limites de tolerância à salinidade, visto que diferentes ambientes poderão ser usados na aquicultura dessa espécie. Em vista disso, os efeitos da salinidade sobre a sobrevivência, osmolaridade plasmática e glicose sanguínea, e parâmetros de desempenho dessa espécie foram avaliados em dois experimentos.

\section{Material e Métodos}

Juvenis de C. undecimalis obtidos no Laboratório de Piscicultura Marinha - Lapmar, UFSC, Florianópolis, SC, foram transportados de avião até Recife, PE. No Laboratório de Piscicultura Marinha, UFRPE, os peixes foram mantidos em tanques circulares de fibra de vidro de $450 \mathrm{~L}$, com a sistemas de recirculação de água.

Os sistemas de recirculação de água foram compostos por um tanque de sedimentação, filtros mecânico e biológico, fracionador de espumas (skimmer) e dois esterilizadores ultravioleta com lâmpadas de 95 W. Os tanques experimentais, o filtro biológico e o skimmer recebiam ar injetado por meio de pedras porosas conectadas a um compressor radial. A temperatura, salinidade e concentração de oxigênio dissolvido foram monitoradas diariamente com um medidor multiparâmetro. As concentrações de amônia e nitrito foram estimadas diariamente com kits comerciais, enquanto a alcalinidade foi medida por titulação.

Com exceção da salinidade, a qual variou conforme o delineamento experimental, as variáveis de qualidade da água não apresentaram diferenças significativas entre os tratamentos nos dois experimentos. A temperatura da água foi mantida entre 27,0 e $29,5^{\circ} \mathrm{C}$ por meio do aquecimento da sala experimental, quando necessário. A concentração de oxigênio dissolvido manteve-se acima de $5,0 \mathrm{mg} / \mathrm{L}( \pm 0,5)$, enquanto as concentrações máximas de amônia total e nitrito foram 0,25 e $0,50 \mathrm{mg} / \mathrm{L}$, respectivamente. Embora varie com a salinidade, a alcalinidade foi mantida acima de $150,0 \mathrm{mg}$ de $\mathrm{CaCO} 3$ por L por meio da adição de bicarbonato de sódio $\left(\mathrm{NaHCO}_{3}\right)$ aos sistemas de recirculação.

Uma ração comercial específica para peixes marinhos contendo 54\% de proteína bruta e $15 \%$ de lipídios foi fornecida duas vezes ao dia (09:00 e 16:00 h) até a saciedade aparente. Restos de ração e resíduos orgânicos foram sifonados diariamente do fundo dos tanques.

\section{Experimento I: Tolerância à salinidade}

Este experimento foi realizado em 15 tanques idênticos aos utilizados na aclimatação, com três repetições cada e 15 peixes por tanque. Peixes com tamanho similar foram selecionados e aclimatizados às condições experimentais por duas semanas. No início do período experimental, o peso e o comprimento total dos robalos eram 88,7 g $( \pm 23,6)$ e $23,5 \mathrm{~cm}( \pm 2,4)$, respectivamente. Após $24 \mathrm{~h}$ de jejum, os peixes foram transferidos diretamente da salinidade 15 para $0,5,15$ (controle), 25 e 35. As diferentes salinidades foram obtidas pela diluição de água do mar com água doce aerada durante $24 \mathrm{~h}$. A alimentação dos peixes e a manutenção das variáveis ambientais foram idênticas ao período de aclimatação.

No início do período experimental (Tempo 0), amostras de sangue foram coletadas de três peixes. Após 4, 24 e $96 \mathrm{~h}$ do choque agudo de salinidade, amostras de sangue também foram coletadas de três peixes por tratamento (um peixe de cada tanque/repetição). Todas as amostras foram obtidas aleatoriamente por punção 
Acta of Fisheries and Aquatic Resources na linha lateral com seringa de $1,0 \mathrm{ml}$ e agulha de 13 x 4,5 mm. Uma alíquota de sangue sem EDTA foi utilizada imediatamente para estimar a glicose com um glicosímetro manual, enquanto outra seringa heparinizada foi utilizada para coletar sangue para a análise da osmolaridade plasmática. As amostras foram refrigeradas e centrifugadas por $8 \mathrm{~min}$ a $4.575 \mathrm{~g}$ para separação do plasma, os quais foram mantidos a $-18^{\circ} \mathrm{C}$. Amostras de água coletadas nos mesmos horários que o sangue também foram mantidas a $-18^{\circ} \mathrm{C}$. $\mathrm{A}$ osmolaridade das amostras de plasma e de água de cada tratamento foi determinada com um osmômetro de pressão a vapor. As análises de sangue foram feitas no Laboratório de Análises Clínicas do Departamento de Farmácia - UFPE, enquanto as medições de osmolaridade foram realizadas no Laboratório de Andrologia, Departamento de Medicina Veterinária, UFRPE.

\section{Experimento II: Crescimento em diferentes salinidades}

Os procedimentos de manejo e alimentação, o monitoramento da qualidade de água e o sistema de manutenção dos peixes foram idênticos aos utilizados no Experimento I. Este estudo, porém, contou com quatro repetições por tratamento, sendo, portanto, utilizados 20 tanques experimentais. Inicialmente, foram selecionados peixes com tamanhos similares, a fim de obter-se uma biomassa próxima em cada unidade experimental. Os peixes foram acondicionados a uma densidade de 15 indivíduos por tanque e aclimatizados por duas semanas a temperatura entre 27 e $29^{\circ} \mathrm{C}$, fotoperíodo natural e aeração constante. A salinidade foi elevada de 15 para 20 com a gradativa adição de água do mar ao sistema de recirculação.

O período experimental teve duração de 45 dias. No início desse período, peixes com médias $( \pm \mathrm{DP})$ de peso e comprimento total de $48,4 \mathrm{~g}( \pm 4,9)$ e $18,7 \mathrm{~cm}( \pm 0,5)$, respectivamente, foram submetidos a um jejum de $24 \mathrm{~h}$, e transferidos diretamente da salinidade de 20 para as salinidades de 0, 10, 20 (controle), 30 e 40. Com exceção da salinidade 40, as demais salinidades foram obtidas como no experimento anterior. A salinidade 40 foi alcançada com a adição de sal marinho sintético à água do mar (salinidade 35).

Após anestesia com óleo de cravo $(50 \mathrm{mg} / \mathrm{L})$, o peso $(\mathrm{g})$ e o comprimento total $(\mathrm{cm})$ de cinco peixes por tanque (20 peixes por tratamento) foram medidos aleatoriamente a cada 15 dias. Ao final do período experimental (dia 45), foram aferidos o peso e o comprimento total de todos os peixes. A sobrevivência (S), ganho de peso (GP), taxa de crescimento específico (TCE), fator de condição (K) e o coeficiente de variação do peso $(\mathrm{CV})$ foram calculados por:

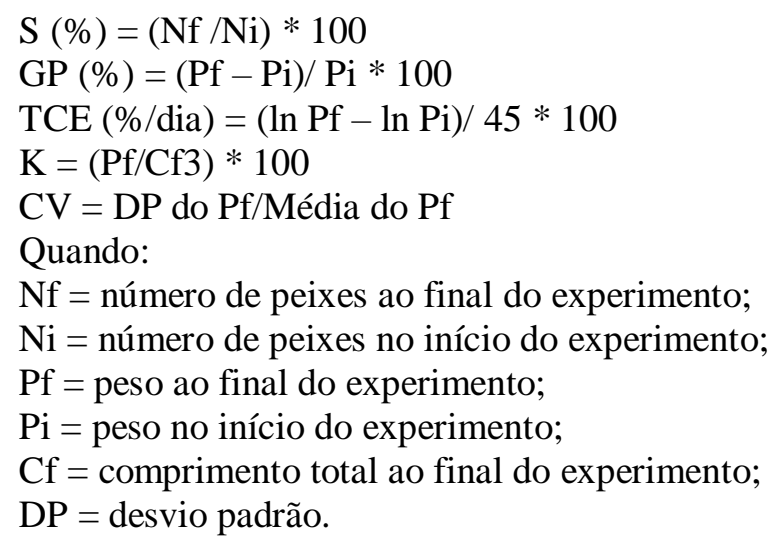

Dois peixes de cada tanque (oito por tratamento) foram aleatoriamente amostrados e o sangue coletado para medir a glicose e a osmolaridade plasmática da mesma forma que no experimento anterior.

\section{Teste estatístico}

Os resultados foram inicialmente submetidos às análises de normalidade e de homogeneidade utilizandose os testes de Shapiro-Wilk e de Bartlett, respectivamente. Posteriormente, utilizou-se a análise de variância (Anova) e o teste de Tukey ( $\alpha=5$ ). Os resultados são apresentados como média \pm desvio padrão (DP).

\section{Resultados}

\section{Experimento I: Tolerância à salinidade}

As salinidades médias da água efetivamente medidas em cada tratamento foram $0,0( \pm 0,0), 5,4( \pm 0,9)$, $14,6( \pm 1,5), 24,2( \pm 1,1)$ e $34,4( \pm 0,5)$, respectivamente. Por outro lado, a osmolaridade da água nas salinidades $0,5,15,25$ e 35 foi $46,4( \pm 16,9), 178,8( \pm 20,2), 428,4( \pm 16,9), 676,8( \pm 15,7)$ e $1.005,8( \pm 8,4) \mathrm{mOsm} / \mathrm{kg}$, respectivamente. 
Acta of Fisheries and Aquatic Resources

A sobrevivência foi $100 \%$ em todos os tratamentos. A concentração média da glicose no sangue dos peixes nos diferentes tratamentos apresentou ampla variação (de 63,7 a 168,3 mg/dL). Não foram observadas diferenças significativas após $0,4,24$ e 96 h do choque de salinidade (Tabela 1). Por sua vez, a osmolaridade do plasma dos peixes apresentou diferenças significativas (Tabela 1). No início do período experimental, a osmolaridade média do plasma era $378,7 \mathrm{mOsm} / \mathrm{kg}$. Entretanto, 4 h após o choque, a osmolaridade dos peixes mantidos na salinidade $35(412,3 \mathrm{mOsm} / \mathrm{kg})$ era maior que a dos peixes nas salinidades 0 e 5 (368,3 e 364,7 $\mathrm{mOsm} / \mathrm{kg}$, respectivamente). Após $96 \mathrm{~h}$, embora a osmolaridade tenha convergido para a faixa de 352-383 $\mathrm{mOsm} / \mathrm{kg}$, diferenças significativas ainda eram observadas entre os peixes na salinidade $5(352,0 \mathrm{mOsm} / \mathrm{kg}) \mathrm{e}$ $25(382,3 \mathrm{mOsm} / \mathrm{kg})$ (Tabela 1$)$.

Tabela 1. Concentração média ( \pm DP) de glicose $(\mathrm{mg} / \mathrm{dL})$ e osmolaridade plasmática $(\mathrm{mOsm} / \mathrm{kg})$ de juvenis de robaloflecha (Centropomus undecimalis) no início do período experimental (Hora 0) e após 4, 24 e 96 h da transferência da salinidade (Sal) 15 para 0, 5, 15, 25 e 35. Letras diferentes indicam diferenças significativas em cada tempo.

\begin{tabular}{cccc}
\hline Hora & Salinidade & Glicose & osmolaridade \\
\hline 0 & 15 & $168,3 \pm 76,6$ & $378,7 \pm 12,7$ \\
\hline & 0 & $157,0 \pm 31,5$ & $368,3 \pm 8,3^{\mathrm{a}}$ \\
& 5 & $89,3 \pm 12,2$ & $364,7 \pm 9,5^{\mathrm{a}}$ \\
4 & 15 & $168,3 \pm 76,6$ & $378,7 \pm 12,7^{\mathrm{ab}}$ \\
& 25 & $121,3 \pm 81,6$ & $399,7 \pm 12,9^{\mathrm{ab}}$ \\
& 35 & $161,0 \pm 100,5$ & $412,3 \pm 26,9^{\mathrm{b}}$ \\
\hline \multirow{3}{*}{24} & 0 & $151,3 \pm 37,1$ & $360,3 \pm 24,6$ \\
& 5 & $101,7 \pm 35,1$ & $384,7 \pm 3,5$ \\
& 15 & $134,3 \pm 51,3$ & $395,0 \pm 26,1$ \\
& 25 & $141,3 \pm 17,8$ & $374,7 \pm 5,7$ \\
& 35 & $92,7 \pm 38,6$ & $380,7 \pm 7,0$ \\
\hline \multirow{2}{*}{96} & 0 & $95,3 \pm 18,0$ & $366,7 \pm 20,8^{\mathrm{ab}}$ \\
& 5 & $63,7 \pm 21,1$ & $352,0 \pm 1,7^{\mathrm{a}}$ \\
& 15 & $99,0 \pm 4,6$ & $371,7 \pm 3,5^{\mathrm{ab}}$ \\
& 25 & $117,0 \pm 36,8$ & $382,3 \pm 3,5^{\mathrm{b}}$ \\
& 35 & $88,7 \pm 8,7$ & $371,3 \pm 3,1^{\mathrm{ab}}$ \\
\hline
\end{tabular}

\section{Experimento II: Crescimento em diferentes salinidades}

Ao final do período experimental de 45 dias, a sobrevivência foi de $100 \%$ em todos os tratamentos.

Assim como no experimento anterior, não foram observadas diferenças significativas nas concentrações médias de glicose sanguínea, mas a osmolaridade plasmática variou significativamente entre os peixes mantidos nas diferentes salinidades (Tabela 2). Ao final do período experimental, a concentração de glicose no sangue variou de 174,5 a $219,9 \mathrm{mg} / \mathrm{dL}$, sendo, mais uma vez, observada uma grande variação nesses resultados, porém sem diferenças significativas. Por sua vez, a osmolaridade foi significativamente mais alta nos peixes criados na salinidade $40(382,4 \mathrm{mOsm} / \mathrm{kg})$ do que nos mantidos em água doce $(346,5 \mathrm{mOsm} / \mathrm{kg})$. As médias de osmolaridade plasmática dos peixes das demais salinidades não diferiram entre si (Tabela 2).

$O$ peso final foi significativamente maior nos peixes criados na salinidade 30 do que nos mantidos nas salinidades 10 e 40, enquanto o peso dos peixes mantidos nas salinidades 0 e 20 não se diferenciaram dos demais (Tabela 2). Por sua vez, o comprimento total foi maior nos indivíduos da salinidade 30 do que nos da salinidade 40. O comprimento dos peixes mantidos nas salinidades 0,10 e 20 não se diferenciaram dos demais.

No início do período experimental, o fator de condição $(\mathrm{K})$ foi estimado em $0,686( \pm 0,010)$, enquanto o coeficiente de variação do peso $(\mathrm{CV})$, em $0,057( \pm 0,023)$. Assim, embora K não tenha variado em relação ao valor inicial, o CV aumentou significativamente ao final do período experimental em todos os tratamentos. Ao final do período experimental não foram observadas diferenças significativas no GP, TCE, K e CV entre os diferentes tratamentos (Tabela 2). 
Acta of Fisheries and Aquatic Resources

Tabela 2. Médias ( $\pm \mathrm{DP})$ da sobrevivência $(\%)$, concentração de glicose (mg/dL), osmolaridade $(\mathrm{mOsm} / \mathrm{kg})$, peso $(\mathrm{g})$, comprimento total (cm), ganho de peso (GP; \%), taxa de crescimento específico (TCE; \%/dia), fator de condição (K), e coeficiente de variação do peso (CV; \%) de juvenis do robalo-flecha (Centropomus undecimalis) após 45 dias de criação nas salinidades $0,10,20,30$ e 40 .

\begin{tabular}{lccccc}
\hline \multicolumn{1}{c}{ Salinidade } & 0 & 10 & 20 & 30 & 40 \\
\hline Sobrevivência & $100 \pm 0$ & $100 \pm 0$ & $100 \pm 0$ & $100 \pm 0$ & $100 \pm 0$ \\
Glicose & $175,4 \pm 71,1$ & $197,6 \pm 14,2$ & $212,0 \pm 64,0$ & $216,8 \pm 60,3$ & $219,9 \pm 29,5$ \\
Osmolaridade & $346,5 \pm 24,1^{\mathrm{a}}$ & $374,1 \pm 14,1^{\mathrm{ab}}$ & $360,9 \pm 3,6^{\mathrm{ab}}$ & $363,8 \pm 12,4^{\mathrm{ab}}$ & $382,4 \pm 13,5^{\mathrm{b}}$ \\
Peso & $62,7 \pm 3,8^{\mathrm{ab}}$ & $58,9 \pm 3,8^{\mathrm{b}}$ & $67,3 \pm 4,1^{\mathrm{ab}}$ & $70,2 \pm 6,9^{\mathrm{a}}$ & $56,9 \pm 5,1^{\mathrm{b}}$ \\
Comprimento & $20,8 \pm 0,4^{\mathrm{ab}}$ & $20,6 \pm 0,4^{\mathrm{ab}}$ & $21,3 \pm 0,4^{\mathrm{ab}}$ & $21,6 \pm 0,6^{\mathrm{a}}$ & $20,3 \pm 0,6^{\mathrm{b}}$ \\
GP & $29,5 \pm 3,4$ & $31,2 \pm 6,1$ & $26,0 \pm 5,0$ & $33,5 \pm 4,7$ & $32,8 \pm 4,7$ \\
TCE & $0,57 \pm 0,05$ & $0,60 \pm 0,10$ & $0,51 \pm 0,08$ & $0,64 \pm 0,07$ & $0,62 \pm 0,07$ \\
K & $0,695 \pm 0,006$ & $0,672 \pm 0,013$ & $0,692 \pm 0,012$ & $0,696 \pm 0,015$ & $0,681 \pm 0,030$ \\
CV & $0,299 \pm 0,029$ & $0,308 \pm 0,027$ & $0,327 \pm 0,040$ & $0,376 \pm 0,088$ & $0,309 \pm 0,054$ \\
\hline
\end{tabular}

\section{Discussão}

Os juvenis de $C$. undecimalis apresentaram alta capacidade adaptativa frente à salinidade. A exposição a variações bruscas de salinidade, como ocorreu nos Experimentos I e II, e a posterior manutenção dos peixes em diferentes salinidades durante um longo prazo de tempo (Experimento II) não resultaram em nenhuma

morte. Estes resultados concordam com os relatos de que $C$. undecimalis é naturalmente encontrada em ambientes que apresentam baixas salinidades ou amplas variações deste parâmetro (Eskinazi, 1972; Chapman et al., 1982; Gilmore et al., 1983; Rivas, 1986; McMichael Jr. et al., 1989; Pérez-Pinzón \& Lutz, 1991; Winner et al., 2010). O ciclo de vida dos robalos do gênero Centropomus compreende a reprodução e o início do desenvolvimento larval em áreas costeiras. Os juvenis, por sua vez, são encontrados em manguezais, estuários e lagunas, onde se abrigam e se alimentam, mas também costumam penetrar nos rios e, ocasionalmente, em

lagoas hipersalinas (Cerqueira, 2005). Alta sobrevivência à exposição ao estresse salino também foram relatadas para $C$. undecimalis (Rocha et al., 2004; Tsuzuki et al., 2007b) e para o linguado Paralychys orbignyanus (Sampaio \& Bianchini, 2002), também uma espécie estuarina-dependente como C. undecimalis.

Além da sobrevivência, os valores plasmáticos de glicose de $C$. undecimalis também não foram afetados pela salinidade em nenhum dos dois experimentos. Embora variações nos níveis de glicose no sangue sejam normalmente observadas em peixes expostos ao estresse (Wendelaar Bonga, 1997; Baldisserotto, 2013), os

índices glicêmicos aqui observados não apresentaram diferenças significativas entre si, apesar de haverem amplas variações em ambos os estudos. Ao transferir juvenis do robalo-peva Centropomus parallelus da salinidade 30 para a água doce, Wosnick (2009) não detectou variações significativas nas concentrações de glicose sanguínea, embora também tenha observado alta variação nos níveis glicêmicos (de 70 a $140 \mathrm{mg} / \mathrm{dL}$ ).

Apesar do presente estudo e o de Wosnick (2009) terem sido realizados com espécies diferentes, embora

filogeneticamente próximas, os resultados concordam em que nem choques agudos de salinidade e nem a manutenção de $C$. undecimalis em diferentes condições salinas durante 45 dias acarretam em respostas secundárias ao estresse.

A redução da salinidade de 30 para 0 fez com que a osmolaridade do plasma em $C$. parallelus variasse entre 305 e 345 mOsm/kg (Wosnick, 2009), o que levou a concluir que essa espécie é capaz de manter a estabilidade extracelular mesmo com a diminuição da salinidade. De modo similar, Pérez-Pinzón \& Lutz (1991) encontraram que, apesar da osmolaridade do plasma de $C$. undecimalis mantidos nas salinidades de 0 a 35 ter aumentado marginalmente com a salinidade, esta variação não foi significativa, uma vez que a osmolaridade se manteve estável entre 352 e $367 \mathrm{mOsm} / \mathrm{kg}$. No Experimento I, não foram detectadas variações na osmolaridade do plasma dos robalos-flecha nas salinidades $0(357,0 \mathrm{mOsm} / \mathrm{kg})$ e $5(358,8 \mathrm{mOsm} / \mathrm{kg})$. Entretanto, as osmolaridades do plasma dos peixes mantidos nessas salinidades foram menores do que as observadas nos peixes mantidos nas salinidades 15, 25 e 35. Gracia-Lopez et al. (2006) também observaram que a redução de salinidade levava à diminuição na osmolaridade plasmática de $C$. undecimalis. Por sua vez, Liebl et al. (2016) relataram a alta sobrevivência de $C$. undecimalis em ambientes de água doce, o que estaria relacionada à capacidade da espécie adaptar-se a diferentes salinidades, alcançando o equilíbrio osmorregulatório rapidamente após mudanças na concentração iônica do ambiente, como foi claramente demonstrado neste estudo. Estes resultados, portanto, confirmam que $C$. undecimalis consegue manter estável a osmolaridade plasmática quando exposto a variações bruscas de salinidade. Por outro lado, Gracia-Lopez et al. (2006) encontraram que $C$. undecimalis mantidos em salinidades entre 0 e 35 apresentaram diferenças significativas na osmolaridade plasmática. As diferenças entre esses estudos podem ser devidas a várias causas, 
Acta of Fisheries and Aquatic Resources incluindo variações no delineamento experimental, espécie-alvo, composição das dietas, duração do período experimental, e/ou tamanho e idade dos peixes. Nesse sentido, Tsuzuki et al. (2007a) ressaltam que a idade dos juvenis de Centropomus pode afetar a tolerância à salinidade. Eles encontraram que juvenis do robalopeva $C$. parallelus com 52 dias após a eclosão (DAE) eram menos tolerantes a transferências abruptas à água doce do que indivíduos com 59 e 73 DAE.

Em relação ao crescimento, embora no Experimento II não tenham sido detectadas diferenças no ganho de peso e nas taxas de crescimento específico, o peso final foi maior na salinidade 30 do que nas salinidades 10 e 40. Alguns estudos relatam maior crescimento em peixes criados em salinidades intermediárias (Woo \& Kelly, 1995; Martinez-Palácios et al., 2004), inclusive em Centropomus (Tsuzuki et al., 2007b). A exposição a salinidades mais baixas por um longo período de tempo pode acarretar na redução do crescimento, possivelmente devido ao aumento do gasto energético associado à osmorregulação, como demonstrado em $P$. orbignyanus (Sampaio \& Bianchini, 2002).

A tolerância à salinidade é de extrema importância para a aquicultura, especialmente a realizada em ambientes estuarinos. Como a salinidade é variável nessas regiões e a osmorregulação é um processo que demanda energia, certas salinidades podem maximizar o crescimento, diminuindo os gastos de energia com a osmorregulação. A inexistência de alterações significativas em grande parte dos parâmetros mensurados neste estudo confirma a capacidade osmorregulatória de $C$. undecimalis e, ao mesmo tempo, sugere a possibilidade de sua criação em ambientes com variadas condições de salinidade.

\section{Conclusões}

O choque agudo de salinidade não afetou a sobrevivência e nem gerou respostas clássicas de estresse, como variações no nível glicêmico, de juvenis do robalo-flecha. Esta espécie demonstrou ser altamente tolerante a variações de salinidade no meio, contando, portanto, com uma ótima capacidade osmorregulatória. O robaloflecha pode ser criado em ambientes de água doce ou marinha e até mesmo os que apresentam amplas variações de salinidade sem reduções significativas de crescimento.

\section{Agradecimentos}

Agradecemos às equipes do Laboratório de Análises Clínicas do Departamento de Farmácia - UFPE, e do Laboratório de Andrologia, Departamento de Medicina Veterinária, UFRPE, pelo indispensável apoio técnico.

\section{Referências}

Alvarez-Lajonchère, L. S., Cerqueira, V. R.; Silva, I. D., Araújo, J. \& Reis, M. A. (2002). Mass production of juveniles of the fat snook Centropomus parallelus in Brazil. Journal of the World Aquaculture Society, 33 : 506-516.

Alvarez-Lajonchère, L. \& Tsuzuki, M. Y. (2008). A review of methods for Centropomus spp. (snooks) aquaculture and recommendations for the establishment of their culture in Latin America. Aquaculture Research, 39: 684-700.

Baldisserotto, B. (2013). Fisiologia de peixes aplicada à piscicultura. Santa Maria: Editora da Universidade Federal de Santa Maria.

Barman, U. K., Jana, S. N., Garg, S. K., Bhatnagar, A. \& Arasu, A. R. T. (2005). Effect of inland water salinity on growth, feed conversion efficiency and intestinal enzyme activity in growing grey mullet, Mugil cephalus (Linn.): Field and laboratory studies. Aquaculture International, 13: 241-256.

Boeuf, G. \& Payan, P. (2001). How should salinity influence fish growth? Comparative Biochemistry and Physiology C, 130(4): 411-423.

Cavalli, R. O. \& Hamilton, S. (2007). Piscicultura marinha no Brasil - Afinal, quais as espécies boas para cultivar? Panorama da Aquicultura, 17(104): 50-55.

Cerqueira, V. R. (2004). Cultivo de peixes marinhos. In: Poli, C. R., Poli, A. T. B., Andreatta, E. R. \& Beltrame, E. (Eds.). Aqüicultura: experiências Brasileiras (pp.369-406). Florianópolis: Multitarefa Editora.

Cerqueira, V. R. (2005). Cultivo do robalo-peva, Centropomus parallelus. In: Baldisserotto, B. \& Gomes, L.C. (Eds.). Espécies Nativas para Piscicultura no Brasil (pp. 403-431), Santa Maria: Editora da UFSM.

Cerqueira, V. R. \& Tsuzuki, M.Y. (2009). A review of spawning induction, larviculture, and juvenile rearing of the fat snook, Centropomus parallelus. Fish Physiology and Biochemistry, 35(1): 17-28.

Chapman, P., Cross, F., Fish, W. \& Jones, K. (1982). Final report for sport fish introductions project. Study 1: Artificial culture of snook. Florida: Florida Game and Fresh Water Fish Commission. 
Acta of Fisheries and Aquatic Resources

Eskinazi, A. M. (1972). Peixes do Canal de Santa Cruz - Pernambuco - Brasil, Trabalhos de Oceanografia da Universidade Federal de Pernambuco, 13(1): 283-302.

Figueiredo, J. L. \& Menezes, N. A. (1980). Manual de peixes marinhos do Sudeste do Brasil. III. Teleostei (2). São Paulo: Museu de Zoologia da Universidade de São Paulo.

Gilmore, R. G., Donohoe, C. J. \& Cooke, D. W. (1983). Observations on the distribution and biology of eastcentral Florida populations of the common snook Centropomus undecimalis (Bloch). Florida Scientist, 46(3-4): 306-313.

Gracia-López, V., Rosas-Vázquez, C. \& Brito-Pérez, R. (2006). Effects of salinity on physiological conditions in juvenile common snook Centropomus undecimalis. Comparative Biochemistry and Physiology A, 145(3): 340-345.

Liebl, F., Amaral-Junior, H., Garcia, S., Souto, L. I. M., Carvalho, C. V. A. \& Cerqueira, V. R. (2016). Desempenho de juvenis de robalo-flecha e robalo-peva submetidos a diferentes densidades de estocagem em água doce. Boletim do Instituto de Pesca, 42(1): 145-155.

Martinez-Palácios, C. A., Morte, J. C., Tello-Ballinas, J. A., Toledo-Cuevas, M. \& Ross, L. G. (2004). The effects of saline environments on survival and growth of eggs and larvae of Chirostoma estor estor Jordan 1880 (Pisces: Atherinidae). Aquaculture, 238: 509-522.

McMichael Jr., R. J., Peters, D. M. \& Parsons, G. R. (1989). Early life history of the snook, Centropomus undecimalis, in Tampa Bay, Florida. Northeast Gulf Science, 10(2): 113-125.

MPA - Ministério da Pesca e Aquicultura. (2013). Boletim Estatístico da Pesca e Aquicultura. Brasil 2011. Brasília: MPA.

Pérez-Pinzón, M. A. \& Lutz, P. (1991). Activity related cost of osmoregulation in the juvenile snook, Centropomus undecimalis. Bulletin of Marine Science, 48(1): 58-66.

Pope, K. L., Blankinship, D. R., Fisher, M. \& Patiño, R. (2006). Status of the common snook (Centropomus undecimalis) in Texas. The Texas Journal of Science, 58(4): 325-332.

Rivas, L. R. (1986). Systematic review of the perciform fishes of the genus Centropomus. Copeia, 1986(3): 579-611.

Rocha, A. J. S., Gomes, V., Ngan, P. V., Passos, M. J. A. C. R. \& Furia, R. R. (2004). Metabolic demand and growth of juveniles of Centropomus parallelus as function of salinity. Journal of Experimental Marine Biology and Ecology, 316: 157-165.

Sampaio, L. A. \& Bianchini, A. (2002). Salinity effects on osmoregulation and growth of the euryhaline flounder Paralichthys orbignyanus. Journal of Experimental Marine Biology and Ecology, 269(2): 187196.

Tsuzuki, M. Y., Cerqueira, V. R., Teles, A. \& Doneda, S. (2007a). Salinity tolerance of laboratory reared juveniles of the fat snook Centropomus parallelus. Brazilian Journal of Oceanography, 55: 1-5.

Tsuzuki, M. Y., Sugai, J. K., Maciel, J. C., Francisco, C. J. \& Cerqueira, V. R. (2007b). Survival, growth and digestive enzyme activity of juveniles of the fat snook (Centropomus parallelus) reared at different salinities. Aquaculture, 271(1-4): 319-325.

Wendelaar Bonga, S. E. (1997). The stress response in fish. Physiological Reviews, 77(3): 591-625.

Winner, B. L., Blewett, D. A., McMichael Jr., R. H. \& Guenther, C. B. (2010). Relative abundance and distribution of common snook along shoreline habitats of Florida estuaries. Transactions of the American Fisheries Society, 139(1): 62-79.

Woo, N. Y. S. \& Kelly, S. P. (1995). Effects of salinity and nutritional status on growth and metabolism of Sparus sarba in a closed seawater system. Aquaculture, 135: 229-238.

Wosnick, N. (2009). Plasticidade osmorregulatória em Centropomus parallelus (Poey, 1860) - Robalo-peva. [Dissertação de Mestrado]. Curitiba (PR): Universidade Federal do Paraná. 